\title{
The missing link between attachment and substance use disorders
}

Volume 4 Issue 3 - 2015

\section{Scott W Stern}

Department of Psychotherapy, Empowering Psychotherapy and Corporate Services, New York, USA

Correspondence: ScottW Stern, Department of Psychotherapy, Empowering Psychotherapy and Corporate Services, New York, USA

Received: November 17, 2015 | Published: November 23, 2015
Are we misdiagnosing primary attachment disorder as a substance use disorder?

Many substance using clients given a primary diagnosis of Substance Use Disorder are slipping through the cracks of the DSM5 when their drug use is actually symptomatic of attachment and separation issues. For many substance abusing clients, their behavior is often symptomatic of co-occurring Attachment Disorder. Clients with a childhood history of an unsuccessful attachment and separation from their primary caregiver often develop Attachment Disorder between infancy and age 3 .

(This should not be mistaken for Reactive Attachment Disorder, a rare and much more serious condition in which infants manifest the inability to attach within a caregiver relationship.)

It is not uncommon for those with this disorder to self medicate feelings of anger, grief, loss, etc. with substances. In doing so, the drug becomes a substitute for the good, reliable caregiver they have felt deprived of since childhood. One might envision the drug as the good mother, the good father, the good spouse in that it is always present and its effects are experienced as reliable--even nurturing.

According to Margaret Mahler's Separation and Individuation process, it would be a healthy process for these clients to "touch back" to the substance (what we call "slips," "brief relapses" or "lapses") as part of Separation and Individuation with a goal of attaining autonomy and independence from the drug.

For this population of Attachment disordered substance users, addiction professionals must be sensitive and supportive of a gradual separation rather than immediate abstinence.

Lewis Carroll depicts this in his coming of age satire, "Alice in Wonderland." Carroll, aka The Reverend Charles Lutwidge Dodgson, was a mathematician, logician, Anglican clergyman, photographer and opium addicted English author. Throughout his book, the child Alice struggles with ambivalence about growing up. At the beginning of the book, she soon discovers substances that alter her size to comfortably fit her circumstances in Wonderland.

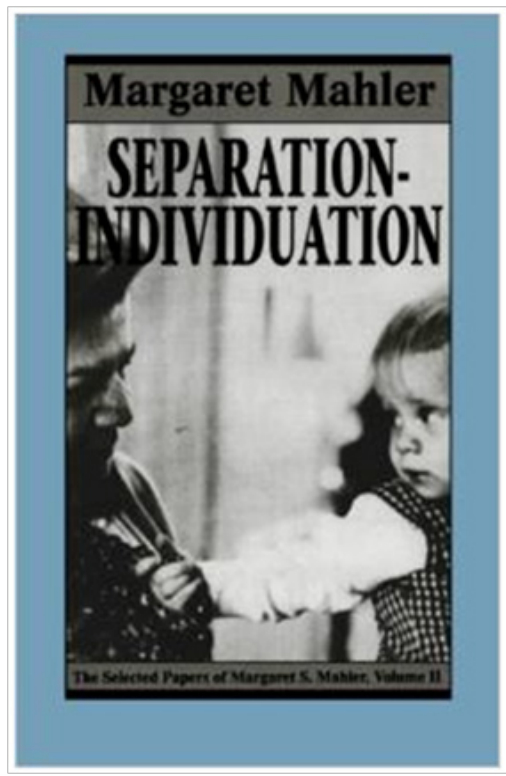

One might say she "touches back" to these substances throughout her adventures to grow up in size or to regress to the smaller sized child Alice until she experiences an independent awakening from a child's Wonderland. Similarly, John Bowlby would concur that many Attachment Disordered substance abusers undergo an attachment process followed by a separation process to the drug as a "good object." Along with this separation from the drug come feelings of grief, anger, ambivalence and so forth.

If so, for the misdiagnosed substance user, the drug becomes a substitute for the good parent, the good caregiver, etc. and "slips" or "lapses" might be part of a natural separation process. 


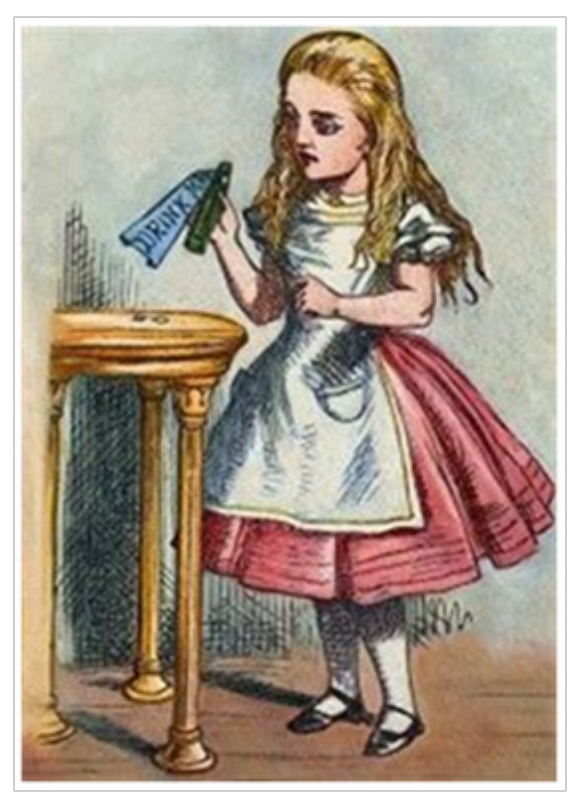

This would mean that abstinence programs would be sending the wrong message by telling the attachment disordered client that "slips" are part of recurring addictive behavior. Being asked to re-count their days of abstinence following a "slip" is shaming and further disrupts a healthy separation and individuation from the drug. This interferes with development of autonomy and independence (see Mahler and Bowlby).

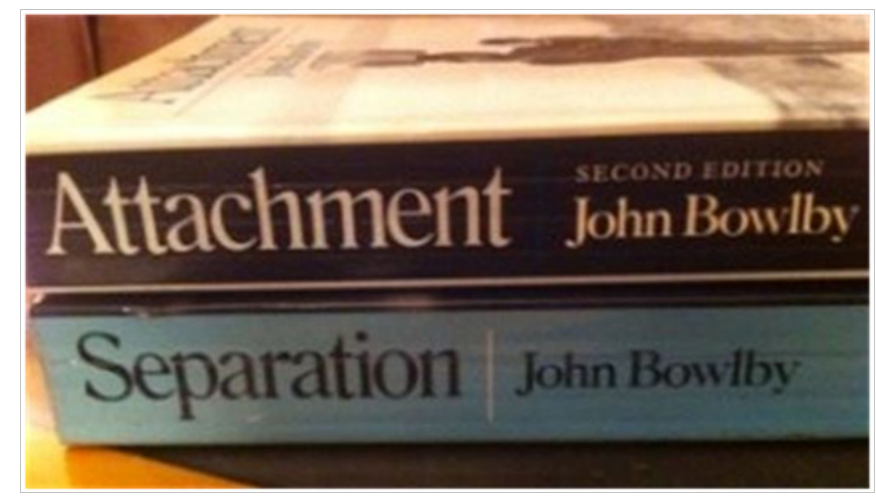

I want to clarify that this applies specifically to individuals who have a primary attachment disorder, and secondary substance use disorder. This understanding also lends itself to the mission of creating a spectrum of treatment approaches to address diverse individual needs. As most of us know, the presenting problem of substance use disorder is not necessarily the client's primary diagnosis.

Therefore, the responsibility falls upon professionals to have a keen understanding of mental health disorders so that they do perform the most comprehensive, personal assessments possible.

For this population, treatment needs to integrate harm reduction and empowerment techniques. This will create a new person-centered approach in the spectrum of behavior modification treatment options.

\section{Acknowledgments}

None.

\section{Conflicts of interest}

Author declares there are no conflicts of interest.

\section{Funding}

None. 